\title{
Influencia de los tratamientos térmicos en recubrimientos anódicos nanotubulares de $\mathrm{TiO}_{2}$
}

\author{
Influence of thermal treatments in nanotubular \\ anodic coatings of $\mathrm{TiO}_{2}$
}

\author{
María Laura Vera ${ }^{1,2}$, Edgard Rubén Henrikson ${ }^{1}$, \\ Hernán Darío Traid ${ }^{1,2}$, Alicia Esther Ares ${ }^{1,2}$, Marta Irene Litter ${ }^{3,4}$
}

\author{
${ }^{1}$ Instituto de Materiales de Misiones, Consejo Nacional de Investigaciones Científicas y Técnicas - Universidad Nacional \\ de Misiones, Colón 1551, Posadas, Misiones, Argentina \\ ${ }^{2}$ Facultad de Ciencias Exactas, Químicas y Naturales, Universidad Nacional de Misiones, Félix de Azara 1552, Posadas, \\ Misiones, Argentina \\ ${ }^{3}$ Gerencia Química, Centro Atómico Constituyentes, Comisión Nacional de Energía Atómica, Av. Gral. Paz 1499, San \\ Martín, Buenos Aires, Argentina \\ ${ }^{4}$ Instituto de Investigación e Ingeniería Ambiental, Universidad Nacional de General San Martín, Campus Miguelete, San \\ Martín, Buenos Aires, Argentina \\ e-mail: lauravera@fceqyn.unam.edu.ar, henriksonedgard@gmail.com, traidhernan@gmail.com, aa- \\ res@fceqyn.unam.edu.ar, litter@cnea.gov.ar
}

\section{RESUMEN}

$\mathrm{El} \mathrm{TiO}_{2}$ es un semiconductor ampliamente utilizado en el proceso de fotocatálisis heterogénea para la descontaminación de aguas. Diversos estudios indican que las estructuras ordenadas de nanotubos en fase anatasa mejoran las propiedades fotocatalíticas del material con respecto a otras morfologías y estructuras de $\mathrm{TiO}_{2}$.

El objetivo del presente trabajo es evaluar la influencia de diferentes tratamientos térmicos sobre la integridad y estructura cristalina de recubrimientos nanotubulares de $\mathrm{TiO}_{2}$ obtenidos mediante la técnica de oxidación anódica.

Para la oxidación se utilizó como electrolito una solución de $0,6 \%$ p/p de fluoruro de amonio y 3,5\% p/p de agua en etilenglicol. Se hizo circular corriente continua a un voltaje constante de $45 \mathrm{~V}$ durante 2 h. Luego de la oxidación anódica, se realizaron tratamientos térmicos de 2 h y 4 h a 450,550 y $650{ }^{\circ} \mathrm{C}$, con rampa de calentamiento de $10^{\circ} \mathrm{C} / \mathrm{min}$. Se caracterizó la morfología y la estructura cristalina de los recubrimientos mediante microscopía electrónica de barrido y difracción de rayos $\mathrm{X}$, respectivamente.

Se obtuvieron recubrimientos nanotubulares de $\mathrm{TiO}_{2}$ de $85 \mathrm{~nm}$ de diámetro interior, $18 \mathrm{~nm}$ de espesor de pared y $7 \mu \mathrm{m}$ de longitud (valores promedio), que mantuvieron su integridad estructural sin cambio en las dimensiones con los tratamientos térmicos aplicados. Sin embargo, la morfología de las paredes de los nanotubos se fue modificando con el tiempo y la temperatura de los tratamientos térmicos, evidenciándose la formación de cristales de anatasa. Incluso a partir de $550^{\circ} \mathrm{C}$ se observaron depósitos nanocristalinos de anatasa sobre la cara exterior de la pared de los nanotubos. En cuanto a la estructura cristalina, los recubrimientos resultaron amorfos previamente a los tratamientos térmicos y cristalinos en fase anatasa luego de los mismos, indicando la estabilización de esta fase incluso hasta $\operatorname{los} 650^{\circ} \mathrm{C}$, temperatura a la que apareció la fase rutilo.

Palabras clave: oxidación anódica, tratamientos térmicos, dióxido de titanio, anatasa, nanotubos.

\section{ABSTRACT}

$\mathrm{TiO}_{2}$ is a semiconductor widely used in heterogeneous photocatalysis processes for water decontamination. Several studies indicate that ordered nanostructures in anatase phase improve the photocatalytic properties with respect to other morphologies and crystal structures of $\mathrm{TiO}_{2}$.

The aim of the present work is to evaluate the influence of different thermal treatments on the structural integrity and crystal structure of the nanotubular $\mathrm{TiO}_{2}$ coatings synthesized by the anodic oxidation technique. 
A solution of $0.6 \% \mathrm{w} / \mathrm{w}$ ammonium fluoride and $3.5 \% \mathrm{w} / \mathrm{w}$ water in ethylene glycol was used as the electrolyte for the oxidation. A direct current was used at a constant voltage of $45 \mathrm{~V}$ during $2 \mathrm{~h}$. After the anodic oxidation, thermal treatments of $2 \mathrm{~h}$ and $4 \mathrm{~h}$ at 450,550 and $650{ }^{\circ} \mathrm{C}$ with a heating ramp of $10^{\circ} \mathrm{C} / \mathrm{min}$ were performed. Morphology and crystalline structure of the coatings were characterized by scanning electron microscopy and X-ray diffraction, respectively.

$\mathrm{TiO}_{2}$ coatings of nanotubes about $85 \mathrm{~nm}$ inner diameter, $18 \mathrm{~nm}$ wall thick and $7 \mu \mathrm{m}$ in length (mean value) were obtained. The nanotubes maintained their structural integrity without changes in the dimensions with the applied thermal treatments. However, the morphology of the walls of the nanotubes was modified with the time and temperature of the thermal treatments, evidencing the formation of anatase crystals. Even on the coatings thermally treated at $550{ }^{\circ} \mathrm{C}$, nanocrystalline anatase deposits on the outside wall of the nanotubes were observed. Regarding the crystal structure, the coatings were amorphous previously to the thermal treatments and were crystalline in anatase phase after thereof, indicating the anatase stabilization even up to $650{ }^{\circ} \mathrm{C}$, temperature at which the rutile phase appeared.

Keywords: anodic oxidation, thermal treatment, titanium dioxide, nanotubes.

\section{INTRODUCCIÓN}

Existe un creciente interés en la síntesis de nanotubos de $\mathrm{TiO}_{2}$, debido al amplio rango de potenciales aplicaciones tecnológicas relevantes de estos materiales, tales como sensores de gases, inmovilización de biomoléculas, células fotovoltaicas, catalizadores térmicos y fotocatalizadores. La fotocatálisis heterogénea empleando $\mathrm{TiO}_{2}$ como catalizador es un proceso avanzado útil para el tratamiento de gases y aguas contaminadas, conteniendo sustancias resistentes a las tecnologías convencionales. En este proceso, se irradia con luz UV una suspensión de $\mathrm{TiO}_{2}$, originándose especies reactivas capaces de transformar los contaminantes [1]. Sin embargo, el uso en el proceso de $\mathrm{TiO}_{2}$ en polvo implica la necesidad de incorporar una costosa etapa de separación y recuperación del catalizador, que puede eliminarse inmovilizando el $\mathrm{TiO}_{2}$ sobre sustratos convenientes. En este sentido, uno de los grandes desafíos tecnológicos es la obtención de recubrimientos de $\mathrm{TiO}_{2}$ de elevada área superficial y actividad fotocatalítica. Diversos estudios indican que las estructuras ordenadas de nanotubos mejoran las propiedades fotocatalíticas del material con respecto a otras morfologías de $\mathrm{TiO}_{2}$ [1].

Los nanotubos de $\mathrm{TiO}_{2}$ pueden ser fabricados por diversas técnicas como sol-gel, anodización, electrodeposición, deposición sonoquímica y métodos que implican el tratamiento químico de partículas de $\mathrm{TiO}_{2}$. Sin embargo, varias de estas técnicas pueden ser complicadas debido a la necesidad del uso de templates o por los procesos químicos involucrados. Entre las técnicas mencionadas, la anodización es una de las más simples y permite obtener arreglos ordenados de nanotubos de $20-90 \mathrm{~nm}$ de diámetro interior y $50 \mathrm{~nm}-1 \mathrm{~mm}$ de largo, dependiendo fundamentalmente de la naturaleza del electrolito y del voltaje empleado [1,2]. Las estructuras nanotubulares se obtienen cuando la oxidación anódica se realiza con electrolitos conteniendo el ion fluoruro $\left(\mathrm{F}^{-}\right)$, debido a reacciones competitivas de formación y disolución del óxido [1-3] La incorporación de compuestos orgánicos al electrolito permite obtener recubrimientos anódicos nanotubulares de varios micrones de longitud [6] y elevados valores de área superficial por unidad de masa y volumen.

En todos los casos, los nanotubos obtenidos anódicamente resultan amorfos y se requiere de tratamientos térmicos a elevadas temperaturas para cristalizarlos en las fases anatasa y/o rutilo, dado que las propiedades del $\mathrm{TiO}_{2}$ y, por lo tanto sus potenciales aplicaciones, dependen de la cristalinidad y de las fases presentes; específicamente, la anatasa es la fase considerada como la más fotoactiva para los procesos de fotocatálisis heterogénea [1]. Por este motivo, resulta de interés evaluar la estabilidad de la fase anatasa así como la integridad estructural y morfológica de los nanotubos obtenidos luego de tratamientos térmicos [1]. Si bien hay varias publicaciones sobre el tema, existen controversias con respecto a la estabilidad estructural de los nanotubos y la temperatura de transición anatasa-rutilo, que fue reportada a $400{ }^{\circ} \mathrm{C}[1], 450{ }^{\circ} \mathrm{C}[1], 500{ }^{\circ} \mathrm{C}$ $[1,2]$ y $600{ }^{\circ} \mathrm{C}[1,2]$. En general, las temperaturas de transición reportadas difieren entre sí debido a que las muestras han sido preparadas por diferentes grupos en diversas condiciones y los nanotubos resultantes son de distintas composiciones y dimensiones características [11].

En este trabajo se presenta la preparación de nanotubos anódicos de $\mathrm{TiO}_{2}$ de $\sim 7 \mu \mathrm{m}$ de longitud, obtenidos a $45 \mathrm{~V}$ en un electrolito con base orgánica de etilenglicol conteniendo $\mathrm{NH}_{4} \mathrm{~F}$ y $\mathrm{H}_{2} \mathrm{O}$, y se evalúa la estabilidad morfológica de los nanotubos y de la fase anatasa con diferentes tratamientos térmicos después del proceso de anodización. 


\section{MATERIALES Y MÉTODOS}

\subsection{Preparación del sustrato}

Como sustrato para realizar las oxidaciones se utilizaron placas de titanio de pureza comercial (Ti cp) de $3 \times$ $2 \mathrm{~cm}^{2}$ y $0,2 \mathrm{~cm}$ de espesor. Las mismas fueron desbastadas en una máquina pulidora (250 rpm) con papeles abrasivos de $\mathrm{SiC}$ de granulometría creciente desde \#240 a \#1500 y pulidas durante $1 \mathrm{~h}$ con pasta de diamante (Praxis) de $1 \mu \mathrm{m}$ lubricada con etilenglicol (Cicarelli). Posteriormente, las placas se limpiaron con agua y detergente, se rociaron con alcohol y se secaron con aire caliente.

\subsection{Oxidación anódica}

La oxidación anódica se realizó a temperatura ambiente, empleando una fuente de corriente continua (JMB LPS360DD). Se utilizaron como cátodo dos láminas de platino, distanciadas a $5 \mathrm{~cm}$ del ánodo de titanio, sumergidas en un recipiente conteniendo el electrolito. Como electrolito se utilizó una solución de 0,6\% p/p de fluoruro de amonio (Biopack) y 3,5\% p/p de agua desmineralizada en etilenglicol (Biopack).

El proceso de oxidación se llevó a cabo a voltaje constante de $45 \mathrm{~V}$ durante $2 \mathrm{~h}$. Las condiciones de anodización se han fijado en base a experiencias previas del grupo que determinaron que $50 \mathrm{~V}$ es el límite de estabilidad estructural de los nanotubos sobre el sustrato de titanio para el electrolito empleado [1,2].

En cada caso, inmediatamente después de la oxidación, las probetas se enjuagaron con agua desmineralizada y se secaron con aire caliente a $50^{\circ} \mathrm{C}$ durante $10 \mathrm{~s}$.

\subsection{Tratamientos térmicos}

Posteriormente a la oxidación anódica se realizaron tratamientos térmicos (TT) de 2 y $4 \mathrm{~h}$ a $450{ }^{\circ} \mathrm{C}$, y de $2 \mathrm{~h}$ a 550 y $650{ }^{\circ} \mathrm{C}$, con rampa de calentamiento de $10^{\circ} \mathrm{C} / \mathrm{min}$ en un horno SIMCIC. En todos los casos, el enfriamiento fue lento dentro del horno.

Las probetas recubiertas se denominaron indicando la temperatura $\left(\right.$ en ${ }^{\circ} \mathrm{C}$ ) y el tiempo (en horas) del tratamiento térmico, ej. T450-t2, para la probeta con TT a $450{ }^{\circ} \mathrm{C}$ durante $2 \mathrm{~h}$. Una probeta anodizada sin tratamiento térmico, se denominó "Sin TT".

\subsection{Caracterización de los recubrimientos}

La observación superficial de los recubrimientos se realizó mediante microscopía electrónica de barrido (MEB, Carl Zeiss Supra 40). Para el análisis de las imágenes, se utilizó el programa ImageJ [1]. En las micrografías MEB de cada recubrimiento, se midió el diámetro interno (Di), el espesor (e) de las paredes y la longitud de los nanotubos. A partir de estos valores, se calculó el área superficial (por unidad de área en $\mathrm{cm}^{2}$ ) de los recubrimientos.

Las fases cristalinas se identificaron mediante difracción de rayos X (DRX) con incidencia rasante de $1^{\circ}$, utilizando un difractómetro Panalytical, modelo Empyrean con detector PIXCEL3D.

\section{RESULTADOS Y DISCUSIÓN}

En la Figura 1 se presentan micrografías de una vista superior de los recubrimientos obtenidos. Puede observarse que los nanotubos se hallan cubiertos por otras nanoestructuras, tipo "nanopastos" [1], que se forman durante oxidaciones prolongadas $(\geq 1 \mathrm{~h})$ en electrolitos orgánicos y se asocian al doblamiento y colapso de los nanotubos por disolución parcial de sus paredes en soluciones orgánicas, como el etilenglicol [4,1]. La presencia de los nanotubos bajo los nanopastos se observa con mayor claridad en el inserto de la Figura 1 (a) correspondiente a la probeta T450-t2. En la mencionada Figura, puede observarse que los nanotubos están abiertos en la parte superior y que los nanopastos que se hallan sobre ellos son más finos y alargados. Sin embargo, en la micrografía de la Figura 1 (b), correspondiente a la probeta T650-t2, se observan nanoestructuras particuladas y fraccionadas, ubicadas sobre los nanotubos, producto del tratamiento térmico a mayor temperatura que por sinterizado produce el colapso de los nanopastos. Adicionalmente, en el inserto de la Figura 1 (b) puede observarse que los nanotubos están cerrados en su extremo inferior.

Si bien existen varias referencias que proponen alternativas para eliminar o evitar la formación de los nanopastos [1-4], estas nanoestructuras podrían resultar beneficiosas cuando los materiales se utilizan como fotocatalizadores heterogéneos dado el aumento en el área superficial y la masa de fotocatalizador. 


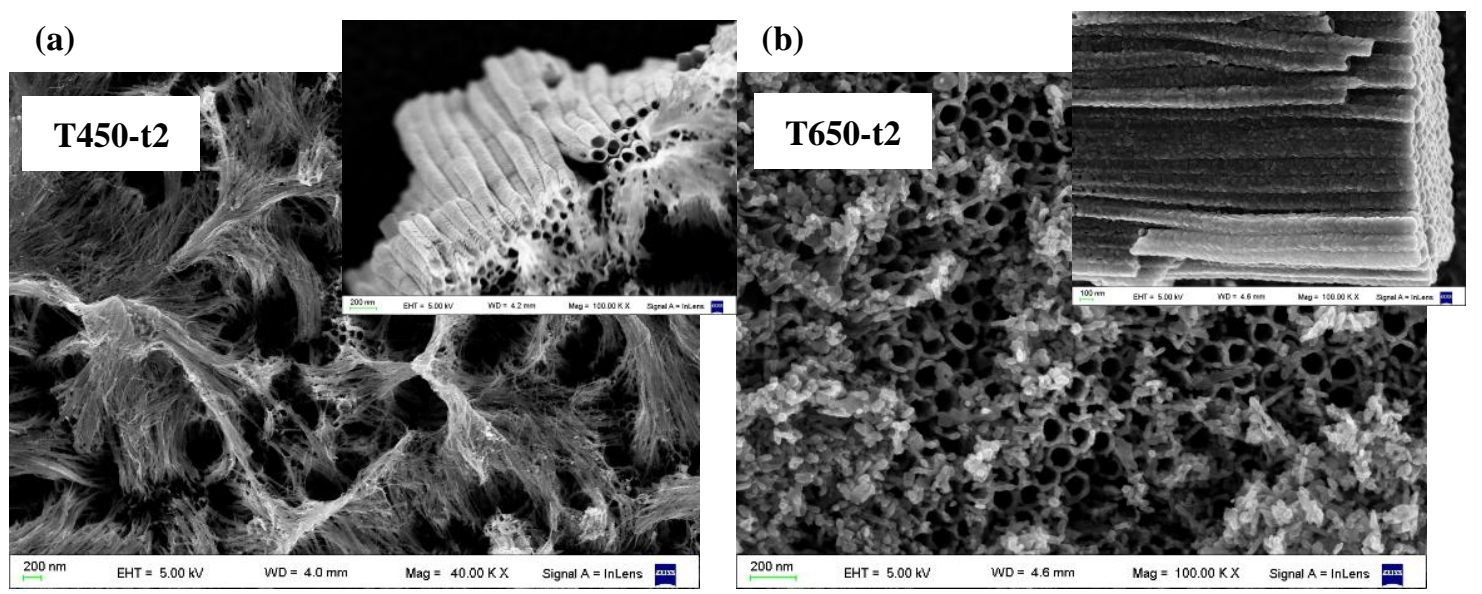

Figura 1: Micrografías electrónicas de barrido de vistas frontales y en perspectiva de los nanotubos.

En la Tabla 1 se presentan las dimensiones características de los nanotubos sin y con tratamientos térmicos. Puede observarse que no hay variación significativa de las dimensiones con los diferentes tratamientos, indicando que las dimensiones de los nanotubos dependen fundamentalmente de las condiciones del anodizado y no de los tratamientos térmicos posteriores. El voltaje determina el diámetro y el espesor de pared de los nanotubos, y el tiempo de anodización influye fundamentalmente en la longitud de los nanotubos [4]. En este trabajo, tanto el voltaje (45 V) como el tiempo de anodización (2 h) se mantuvieron constantes en todas las probetas.

Los valores de la Tabla 1 indican que no habría reducción del área superficial como resultado de efectos de sinterizado asociado a los tratamientos térmicos realizados que provoquen colapso de la estructura nanotubular. De acuerdo a los cálculos realizados, los valores de área superficial oscilan entre los 129 y los $206 \mathrm{~cm}^{2} / \mathrm{cm}^{2}$.

Tabla 1: Dimensiones características de los nanotubos y área por unidad de superficie de los recubrimientos.

\begin{tabular}{c|c|c|c|c}
\hline MUESTRAS & $\begin{array}{c}\text { DIÁMETRO } \\
\text { INTERIOR [nm] }\end{array}$ & $\begin{array}{c}\text { ESPESOR DE } \\
\text { PARED [nm] }\end{array}$ & LONGITUD $[\boldsymbol{\mu m}]$ & $\begin{array}{c}\text { ÁREA POR cm } \\
{\left[\mathbf{c m}^{2}\right]}\end{array}$ \\
\hline Sin TT & $87 \pm 8$ & $17 \pm 3$ & $6 \pm 1$ & 152 \\
\hline T450-t2 & $85 \pm 7$ & $17 \pm 2$ & $8 \pm 1$ & 206 \\
\hline T450-t4 & $84 \pm 8$ & $19 \pm 2$ & $7 \pm 1$ & 175 \\
\hline T550-t2 & $85 \pm 8$ & $17 \pm 3$ & $5 \pm 1$ & 129 \\
\hline T650-t2 & $86 \pm 6$ & $18 \pm 3$ & $7 \pm 1$ & 176 \\
\hline
\end{tabular}

En la Figura 2 se observa que los difractogramas de todos los recubrimientos presentan picos correspondientes al sustrato de Ti y a la fase anatasa, indicando que con los diferentes tratamientos térmicos realizados la anatasa es la fase estable incluso hasta los $650^{\circ} \mathrm{C}$. Sin embargo, en el difractograma correspondiente a la muestra T650-t2, se observa un pequeño pico en $2 \theta \sim 27,46^{\circ}$ (marcado con un recuadro en la Figura 2), que indicaría la aparición de la fase rutilo [1].

Como se ha mencionado previamente en la Sección 1 (Introducción) hay diversos reportes de temperaturas de transición anatasa-rutilo para los nanotubos anódicos de $\mathrm{TiO}_{2}$ soportados, en su mayoría inferiores a $\operatorname{los} 600^{\circ} \mathrm{C}[10-15]$. 


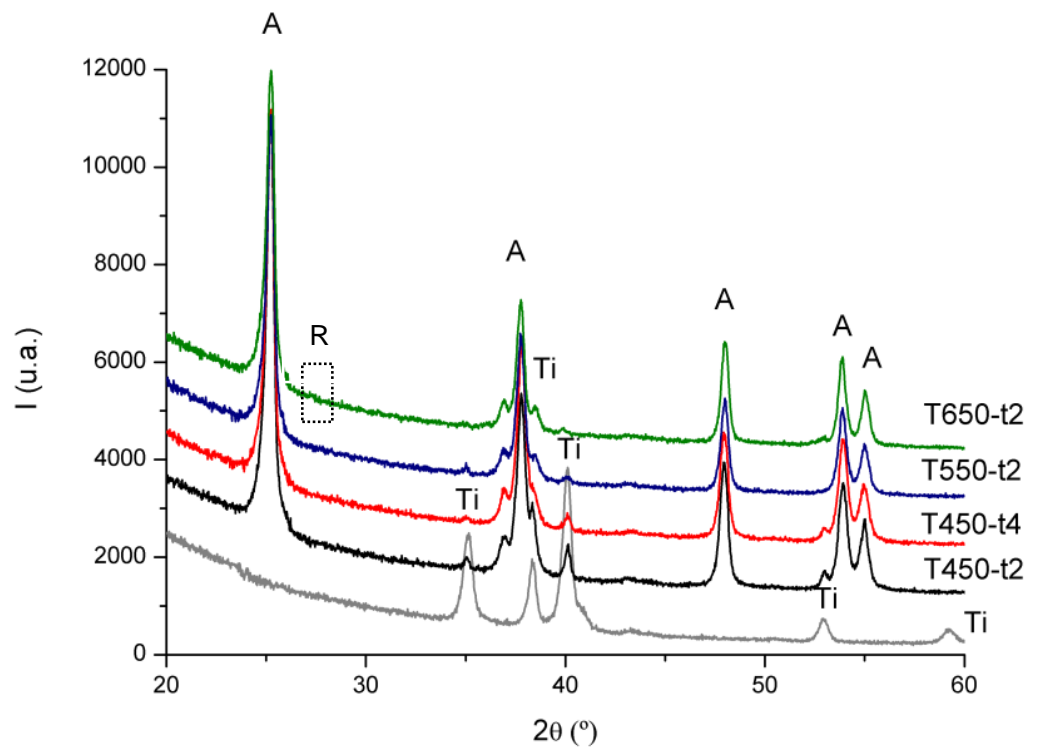

Figura 2: Difractogramas de rayos $\mathrm{X}$ del sustrato de Ti y de los recubrimientos estudiados. Los picos indexados con $\mathrm{A}$ corresponden a la fase anatasa, con $\mathrm{R}$ a la fase rutilo y con $\mathrm{Ti}$ al titanio.

En la Figura 3 se presentan vistas laterales de los nanotubos. En la Figura 3 (a), correspondiente a los nanotubos sin TT, se observa la presencia de estrías que rodean los nanotubos, producto del contenido de agua en la solución del electrolito (superior a 0,5\%) [1]que promueve la evolución de oxígeno durante la formación de los nanotubos [24]. Esta morfología de las paredes no cambia con el tratamiento a $450{ }^{\circ} \mathrm{C}$, como puede observarse en la Figura 3 (b). En la Figura 3 (c), correspondiente a una probeta con tratamiento térmico más prolongado a la misma temperatura, se observan líneas similares a bordes de grano unidos unos con otros en las paredes de los nanotubos, producto de la formación de los cristales de anatasa [9,1]. En la Figura 3 (d), correspondiente a los nanotubos tratados a $550{ }^{\circ} \mathrm{C}$, se observa que los cristales crecieron como protuberancias, no sólo en la pared de los nanotubos, sino sobre ella. En la Figura 3 (e), correspondiente a los nanotubos tratados a $650{ }^{\circ} \mathrm{C}$, ya pueden distinguirse cristales individuales de anatasa de $\sim 70 \mathrm{~nm}$ (valor promedio).

Esta evolución de la morfología de la pared de los nanotubos es demostrativa del proceso de nucleación y crecimiento por el cual se da primero la transformación de fase amorfa a anatasa y, cuando los cristales de anatasa alcanzan un tamaño crítico, se produce la transformación de anatasa a rutilo [11]. VARGHESE et al. [9] sostienen que la transición a rutilo sería coincidente con el completo colapso de la estructura nanotubular, hecho no observado en nuestro caso hasta los $650{ }^{\circ} \mathrm{C}$, pero que podría darse a mayores temperaturas con el avance de la transformación a la fase rutilo.

Cabe aclarar que en todas las micrografías se observan nanotubos de diferente longitud e incluso quebrados, lo que se debería a que esta vista se obtuvo rasgando los recubrimientos, y no a la pérdida de integridad estructural como resultado de los TT. 
(a)

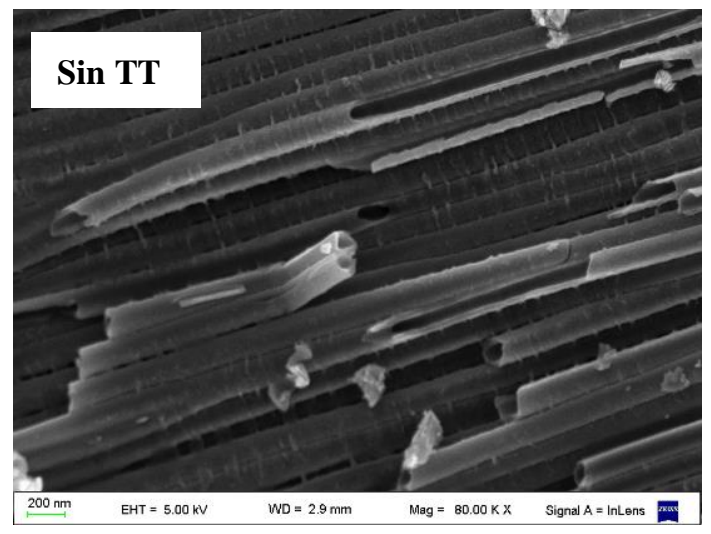

(b)

(c)

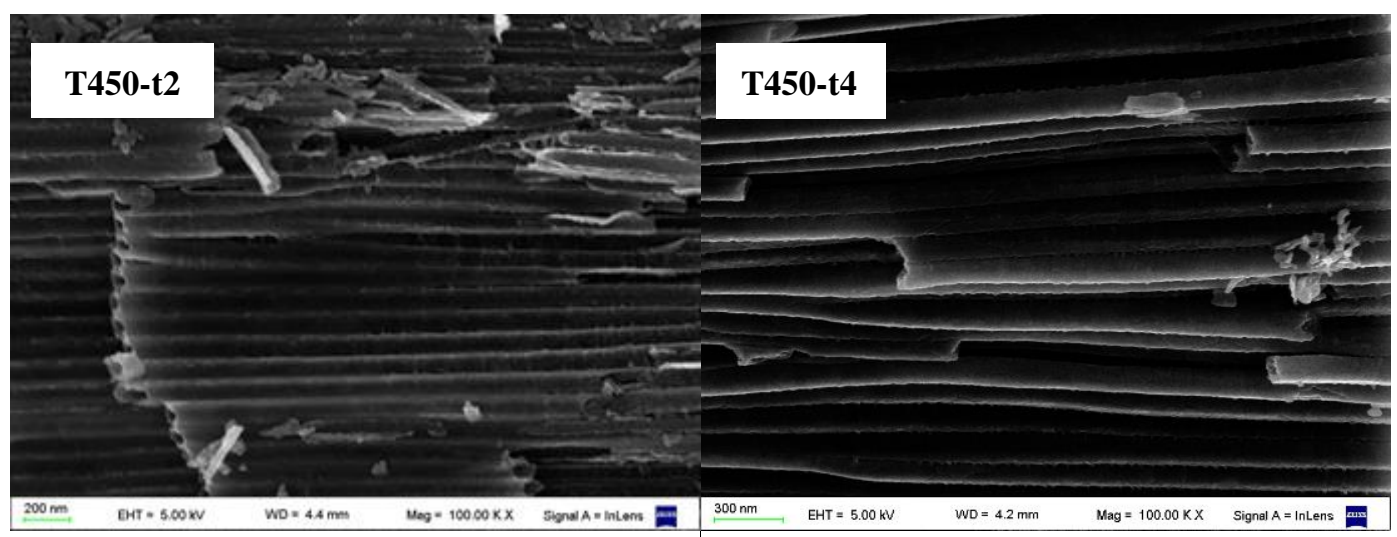

\begin{tabular}{l|l} 
(d) & (e)
\end{tabular}

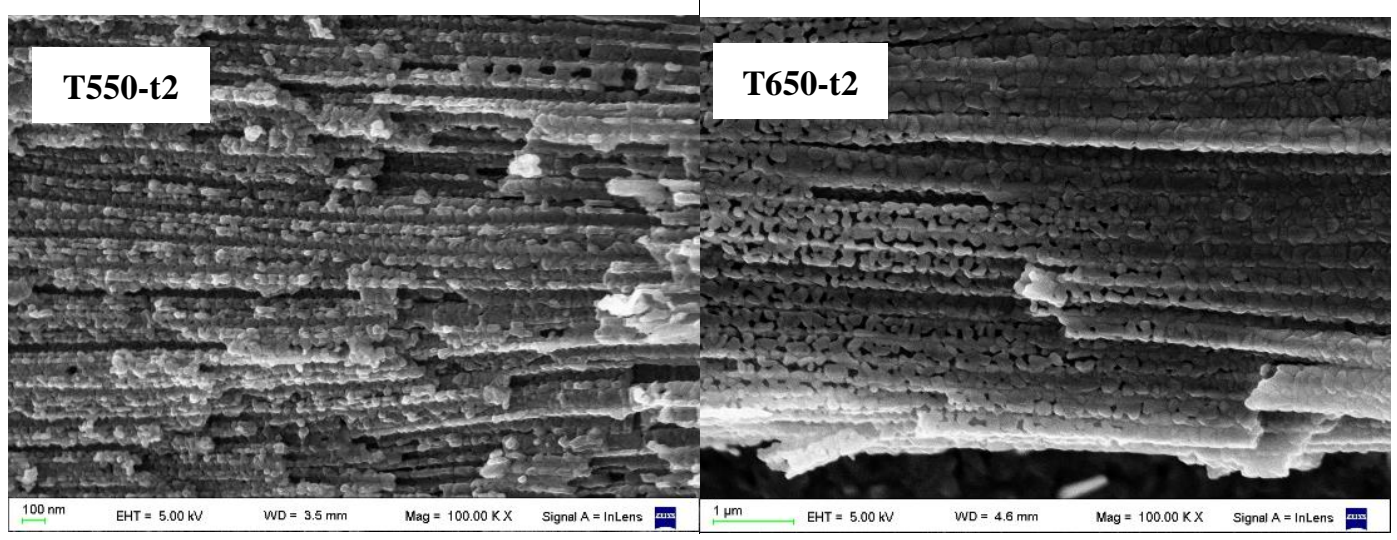

Figura 3: Micrografías electrónicas de barrido de vistas laterales de los nanotubos con diferentes tratamientos térmicos.

\section{CONCLUSIONES}

Se obtuvieron nanotubos de $\mathrm{TiO}_{2}$ de $\sim 85 \mathrm{~nm}$ de diámetro interior, $\sim 18 \mathrm{~nm}$ de espesor de pared y $\sim 7 \mu \mathrm{m}$ de longitud que resultaron cristalinos en fase anatasa después de los tratamientos térmicos, incluso hasta $650{ }^{\circ} \mathrm{C}$, temperatura a partir de la cual aparece la fase rutilo, sin cambios en las dimensiones características y manteniendo su integridad estructural, pero con cambios en la morfología de las paredes de los nanotubos, producto de la formación y crecimiento de los cristales de anatasa y posterior transformación a rutilo. Para estos nanotubos preparados con $0,6 \% \mathrm{p} / \mathrm{p}$ de fluoruro de amonio y 3,5\% p/p de agua en etilenglicol, a $45 \mathrm{~V}$ durante $2 \mathrm{~h}$, la temperatura límite superior para cristalizarlos en fase anatasa sin pérdida estructural sería $650{ }^{\circ} \mathrm{C}$ de cara a emplearlos como fotocatalizadores soportados para el tratamiento de aguas o aire.

\section{AGRADECIMIENTOS}

Los autores agradecen al Consejo Nacional de Investigaciones Científicas y Técnicas (CONICET) y a la 
Agencia Nacional de Promoción Científica y Tecnológica (ANPCyT) de Argentina por el financiamiento de la presente investigación y al Dr. Daniel Vega del Laboratorio de Difracción de Rayos X, Departamento Física de la Material Condensada, Gerencia de Investigación y Aplicaciones, Comisión Nacional de Energía Atómica de Argentina. E. R. Henrikson agradece al Comité Ejecutivo de Desarrollo e Innovación Tecnológica (CEDIT) de la Provincia de Misiones, Argentina, por una beca de Iniciación a la Investigación.

\section{BIBLIOGRAFÍA}

[1] SCHNEIDER, J., BAHNEMANN, D., YE, J., et al., Photocatalysis: Fundamentals and Perspectives, 1 ed., Cambridge, The Royal Society of Chemistry, 2016.

[2] NAKATA, K., FUJISHIMA, A., "TiO 2 photocatalysis: design and applications", Journal of Photochemistry and Photobiology C: Photochemistry, v. 13, n. 3, pp. 169-189, 2012.

[3] GRIMES, C.A., MOR, G.K., TiO 2 Nanotube Array, Synthesis, Properties, and Applications, 1 ed., New York, Springer-Verlag, 2009.

[4] REGONINI, D., BOWEN, C.R., JAROENWORALUCK, A., et al., "A review of growth mechanism, structure and crystallinity of anodized $\mathrm{TiO}_{2}$ nanotubes", Materials Science and Engineering R, v. 74, n. 12, pp. 377-406, 2013.

[5] PARAMASIVAM, I., JHA, H., LIU, N., et al., "A review of photocatalysis using self-organized $\mathrm{TiO}_{2}$ nanotubes and other ordered oxide nanostructures", small, v.8, n.20, pp.3073-3103, 2012.

[6] OH, H-J., KIM, I-K., JANG, K-W., et al., "Influence of electrolyte and anodic potentials on morphology of titania nanotubes", Metals and Materials International, v. 18, n. 4, pp. 673-677, 2012.

[7] WANG, Y., WU, Y., QIN, Y., et al., "Rapid anodic oxidation of highly ordered $\mathrm{TiO}_{2}$ nanotube arrays", Journal of Alloys and Compounds, v. 509, pp. L157-L160, 2011.

[8] FUJISHIMA, A., ZHANG, X., "Titanium dioxide photocatalysis: present situation and futures approaches", Comptes Rendus Chimie, v. 9, n. 5, pp. 750-760, 2006.

[9] VARGHESE, O.K., GONG, D., PAULOSE, M., et al., "Crystallization and high-temperature structural stability of titanium oxide nanotube arrays”, Journal of Materials Research, v. 18 pp. 156-165, Jan. 2003.

[10] YANG, Y., WANG, X.H., LI, L.T., "Synthesis and photovoltaic application of high aspect-ratio $\mathrm{TiO}_{2}$ nanotube arrays by anodization”, Journal of the American Ceramic Society, v. 91, pp. 632-635, 2008.

[11] FANG, D., LUO, Z., HUANG, K., et al., "Effect of heat treatment on morphology, crystalline structure and photocatalysis properties of $\mathrm{TiO}_{2}$ nanotubes on Ti substrate and freestanding membrane", Applied Surface Science, v. 257, pp. 6451-6461, 2011.

[12] REGONINI, D., JAROENWORALUCK, A., STEVENS, R., et al., "Effect of heat treatment on the properties and structure of $\mathrm{TiO}_{2}$ nanotubes: phase composition and chemical composition", Surface and Interface Analysis, v. 42, pp. 139-144, 2010.

[13] JAROENWORALUCK, A., REGONINI, D., BOWEN, C.R., et al., "A microscopy study of the effect of heat treatment on the structure and properties of anodised $\mathrm{TiO}_{2}$ nanotubes", Applied Surface Science, v. 256, pp. 2672-2679, 2010.

[14] LI, G., LIU, Z.Q., LU, J., et al., "Effect of calcination temperature on the morphology and surface properties of $\mathrm{TiO}_{2}$ nanotube arrays", Applied Surface Science, v. 255, pp. 7323-7328, 2009.

[15] YU, J.G., WANG, B., "Effect of calcination temperature on morphology and photoelectrochemical properties of anodized titanium dioxide nanotube arrays", Applied Catalysis B: Environmental, v. 94, pp. 295-302, 2010.

[16] HENRIKSON, E., VERA, M.L., TRAID, H., et al., "Fabricación de recubrimientos nanotubulares de $\mathrm{TiO}_{2}$ y evaluación de su eficiencia fotocatalítica", In: Congreso Nacional de Estudiantes de Ingniería Química (CONEIQ), San Juan, Set. 2015.

[17] HENRIKSON, E., VERA, M.L., TRAID, H., et al., "Fabricación de recubrimientos nanotubulares de dióxido de titanio", In: Actas del $5^{\circ}$ Encuentro de Jóvenes Investigadores en Ciencia y Tecnología de Materiales, 712-G, pp. 373-376, Tandil, Oct. 2015.

[18] SCHNEIDER, C.A., RASBAND, W.S., ELICEIRI, K.W., "NIH Image to ImageJ: 25 years of image analysis", Nature Methods, v. 9, pp. 671-675, 2012.

[19] BERGER, S., HAHN, R., ROY, P., et al., "Self-organized $\mathrm{TiO}_{2}$ nanotubes: Factors affecting their mor- 
phology and properties", Physica Status Solidi (b), v. 247, n. 10, pp. 2424-2435, Sep. 2010.

[20] YASUDA, K., MACAK, J.M., BERGER, S., et al., "Mechanistic aspects of the self-organization process for oxide nanotube formation on valve metals", Journal of The Electrochemical Society. v. 154, n. 9 pp. C472-C478, Jun. 2007.

[21] KIM, D., GHICOV, A., SCHMUK, P., "TiO 2 Nanotube arrays: Elimination of disordered top layers ("nanograss") for improved photoconversion efficiency in dye-sensitized solar cells", Electrochemistry Communications, v. 10, pp. 1835-1838, 2008.

[22] PAULOSE, M., PRAKASAM, H., VARGHESE, O., et al., " $\mathrm{TiO}_{2}$ nanotube arrays of 1000 um length by anodization of titanium foil: phenol red diffusion", The Journal of Physical Chemistry C, v. 111, pp. 1499214997, Sep. 2007.

[23] REGONINI, D., SATKA, A., ALLSOPP, D.W.E., et al., "Anodised titania nanotubes prepared in a glycerol/NaF electrolyte", Journal of Nanoscience and Nanotechnology, v. 9, pp. 4410-4416, 2009.

[24] SO, S., K. LEE, P. SCHMUKI, "Ultrafast growth of highly ordered anodic $\mathrm{TiO}_{2}$ nanotubes in lactic acid electrolytes", Journal of the American Chemical Society, v. 134, pp. 11316-11318, 2012.

[25] INTERNATIONAL Centre for Diffraction Data, Joint Committee on Powder Diffraction Standards, JCPDS 21-1276.

[26] RAJA, K.S., GANDHI, T., MISRA, M., "Effect of water content of ethylene glycol as electrolyte for synthesis of ordered titania nanotubes", Electrochemistry Communications, v. 9, pp. 1069-1076, May. 2007.

[27] ALBU, S.P., TSUCHIYA, H., FUJIMOTO, S., SCHMUKI, P., "TiO ${ }_{2}$ nanotubes - Annealing effects on detailed morphology and structure", European Journal of Inorganic Chemistry. pp. 4351-4356, 2010. 